\title{
Resveratrol inhibits foam cell formation via NADPH oxidase 1- mediated reactive oxygen species and monocyte chemotactic protein-1
}

\author{
Dae-Weon Park ${ }^{1 *}$, Kheewoong Baek ${ }^{3 *}$, \\ Jae-Ryong Kim ${ }^{1}$, Jae-Jin Lee ${ }^{2}$, \\ Sang-Ho Ryu' ${ }^{2}$, Byung-Rho Chin ${ }^{2}$ \\ and Suk-Hwan Baek ${ }^{1,4}$ \\ ${ }^{1}$ Aging-Associated Vascular Disease Research Center \\ Department of Biochemistry and Molecular Biology \\ ${ }^{2}$ Department of Dentistry \\ College of Medicine, Yeungnam University \\ Daegu 705-717, Korea \\ ${ }^{3}$ Hankuk Academy of Foreign Studies \\ Youngin 449-854, Korea \\ ${ }^{4}$ Corresponding author: Tel, 82-53-620-4523; \\ Fax, 82-53-623-8032; E-mail, sbaek@med.yu.ac.kr \\ *These authors contributed equally to this work. \\ DOI 10.3858/emm.2009.41.3.020
}

Accepted 18 November 2008

Abbreviations: FoxO, forkhead box O; MCP-1, monocyte chemoattractant protein-1; NAC, N-acetylcysteine; Nox, NADPH oxidase; ROS, reactive oxygen species

\begin{abstract}
Resveratrol is a polyphenolic compound in red wine that has anti-oxidant and cardioprotective effects in animal models. Reactive oxygen species (ROS) and monocyte chemotactic protein-1 (MCP-1) play key roles in foam cell formation and atherosclerosis. We studied LPS-mediated foam cell formation and the effect of resveratrol. Resveratrol pretreatment strongly suppressed LPS-induced foam cell formation. To determine if resveratrol affected the expression of genes that control ROS generation in macrophages, NADPH oxidase 1 (Nox1) was measured. Resveratrol treatment of macrophages inhibited LPS-induced Nox1 expression as well as ROS generation, and also suppressed LPS-induced MCP-1 mRNA and protein expression. We investigated the upstream targets of Nox1 and MCP-1 expression and found that Akt-forkhead transcription factors of the $\mathrm{O}$ class (FoxO3a) is an important signaling pathway that regulates both genes. These inhibitory effects of resveratrol on Nox1 expression and MCP-1 production may target to the Akt
\end{abstract}

and FoxO3a signaling pathways.

Keywords: atherosclerosis; CCL2 protein; foam cells; FoxO3a protein; NADPH oxidase 1; reactive oxygen species; resveratrol

\section{Introduction}

Resveratrol is a polyphenol existing in grapes, berries, and peanuts. Resveratrol protects plants from fungal infection; exhibits anti-oxidative, antiinflammatory, and chemopreventive properties in mammalian systems (Park et al., 2007); and inhibits macrophage activation (Pervaiz, 2003). In addition, resveratrol has been proposed to contribute to anti-atherosclerotic properties (Hou et al., 2008). A number of studies using animal models support this suggestion (Zang et al., 2006). Mechanisms proposed to explain the anti-atherosclerotic properties of resveratrol include reduction of LDL oxidation, inhibition of monocyte chemotactic protein-1 (MCP-1) production (Cullen et al., 2007a), inhibition of macrophage inflammation, and control of plasma cholesterol levels. However, the precise mechanisms concerning the anti-atherosclerosis effects of resveratrol remain unclear.

Oxidative stress-mediated LDL modification has a key role in initiation of the atherosclerotic process (Carnevale et al., 2007). The production of superoxide anion contributes to the pathology of foam cell formation and atherosclerosis by promoting conversion from LDL to oxLDL. Several kinds of cells, including macrophages, endothelial cells, and smooth muscle cells are likely to play a role in generating oxLDL. MCP-1 is also considered one of the most important chemokine-related factors in the process of foam cell formation. LPS controls macrophage function by producing MCP-1, with reactive oxygen species (ROS) being the most important intermediate (Fontaine et al., 2007). The LPS-mediated ROS production mechanism is very diverse and includes NADPH oxidase (Nox) and mitochondria respiratory chain systems. However, even though the production of ROS is considered one of the most important factors controlling MCP-1 production, the precise nature of the control is unclear. 
The most important ROS source in the vascular system is Nox (Bedard and Krause, 2007). Noxes are a family of multi-subunit enzymes. Recently, several Nox isoforms have been reported (Bedard and Krause, 2007), which mainly differ in their large membrane-bound Nox subunit. Of the six proteins hitherto described, Nox1, Nox2, and Nox4 are involved in vascular ROS formation (Schroder et al., 2007). In the vascular system, Nox1 is expressed in vascular smooth muscle cells; Nox2 in macrophages, adventitial fibroblasts, and to some extent also in endothelial cells; and Nox4 in endothelial and smooth muscle cells. ROS that causes LDL conversion is produced in various routes. LDL oxidation in macrophages is known to depend on ROS produced usually from Nox2 (Bedard and Krause, 2007). Recently, the importance of different kinds of Nox in macrophages was suggested (Takeya and Sumimoto, 2006), but research on Nox1 has been scant. Nox1 was the first homolog of the Nox family found in nonmyeloid cells (Gaggioli et al., 2007). It shares 58\% identity with the Nox2 and is expressed mainly in the colon epithelium. The function of Nox1 may similarly be in host defense (Banfi et al., 2000). Other functions of Nox 1 have been recently reported as well. For example, Nox1 knockout mice decreases hypertension by angiotensin II, and exhibits protection effects from aortic dissection. However, there has not been any report that discusses the function of Nox1 related to MCP-1 production or foam cell formation. Resveratrol reduces Nox activity in rat aortic homogenate and macrophages (Chow et al., 2007), however, it is unknown which kind of Nox activation resveratrol inhibits. We therefore sought to examine how resveratrol regulates macrophage ROS production and to examine if such regulation correlates with an effect on the functional MCP-1 secretion and foam cell formation of the macrophages.

The purpose of this study was to investigate whether LPS can induce Nox1 expression and to determine the signaling mechanisms responsible. We also investigated whether resveratrol can inhibit LPS-induced Nox1 and MCP-1 expression. Our results demonstrate that LPS is a potent inducer of Nox 1 and MCP-1 expression in mouse macrophages via an Akt and forkhead transcription factors of the $\mathrm{O}$ class (FoxO3a)-dependent signaling pathway, and that this pathway is inhibited by resveratrol.

\section{Results}

Resveratrol blocks LPS-induced foam cell formation

We examined the influence of resveratrol in foam cell formation, which is an important marker in the early stages of atherosclerosis. We added LPS or LDL with LPS, which can induce foam cell formation in cultured macrophages. Foam cell formation converted from macrophages was analyzed through Oil-red O staining. LDL weakly influenced formation of foam cells, while LDL with LPS treated in macrophages together resulted in the typical formation of foam cells. We next examined the effects of resveratrol in foam cell formation induced by LPS. We pretreated $2.5 \mu \mathrm{M}$ of resveratrol for 1 $\mathrm{h}$, treated with LPS and LDL continually for $24 \mathrm{~h}$, and checked the formation of foam cells through Oil-red $\mathrm{O}$ staining. Resveratrol effectively inhibited foam cell formation induced by LPS (Figure 1).

\section{Resveratrol suppresses LPS-induced ROS generation and Nox1 expression}

Given the well-documented anti-oxidative mechanisms of resveratrol, we focused on the influence of ROS production in resveratrol-mediated inhibition of foam cell formation. As seen in Figure 2A, LPS strongly induced the production of ROS, and such production was blocked by pretreatment of antioxidant $\mathrm{N}$-acetyl cysteine (NAC) or apocynin. These results suggest the importance of ROS production caused by LPS in the conversion process of foam cell production in macrophages, and especially, the inhibition effects of ROS production by apocynin suggests a possibility of Nox activity as well (Vejrazka et al., 2005). We tested the inhibitory effects of ROS production by resveratrol using a lucigenin assay; pretreatment of resveratrol
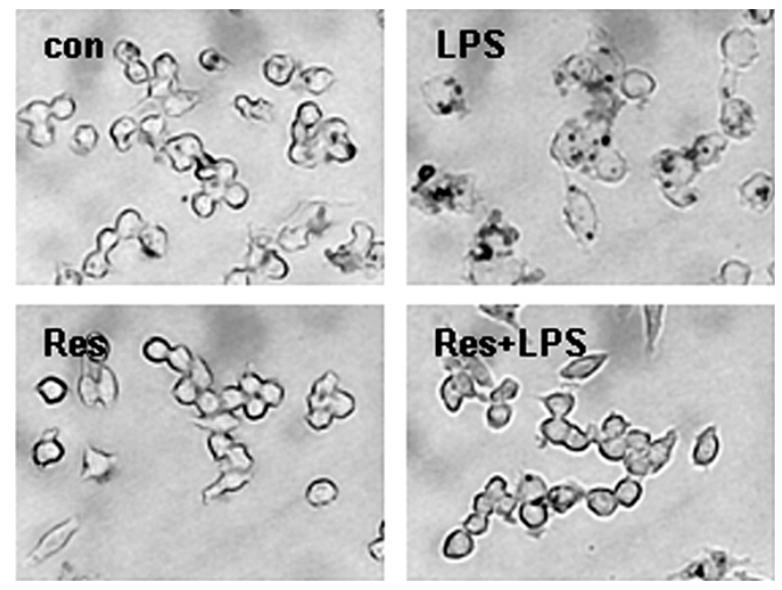

Figure 1. Resveratrol inhibits LPS-induced foam cell formation. Raw264.7 macrophages were cultured in the absence or presence of resveratrol with LPS $(100 \mathrm{ng} / \mathrm{ml})$ containing LDL $(50 \mu \mathrm{g} / \mathrm{ml})$ for $24 \mathrm{~h}$, stained with Oil-red $O$ to label intracellular lipid droplets, and then photographed at a magnification of $\times 400$. 
A

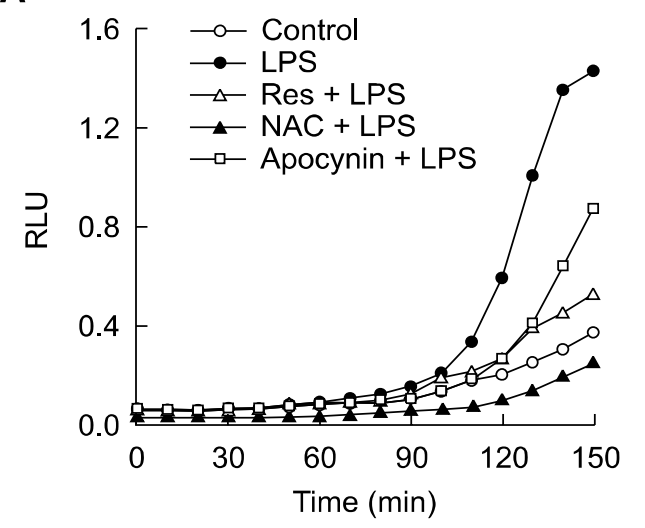

B
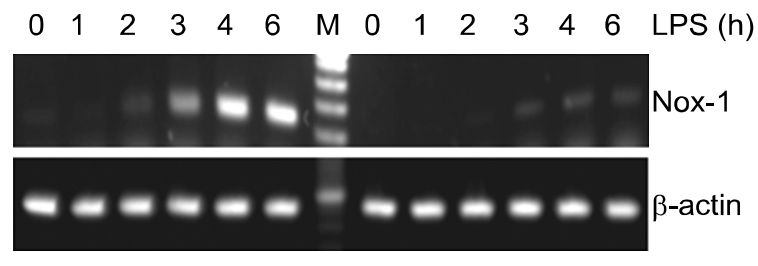

Control

Resveratrol

C

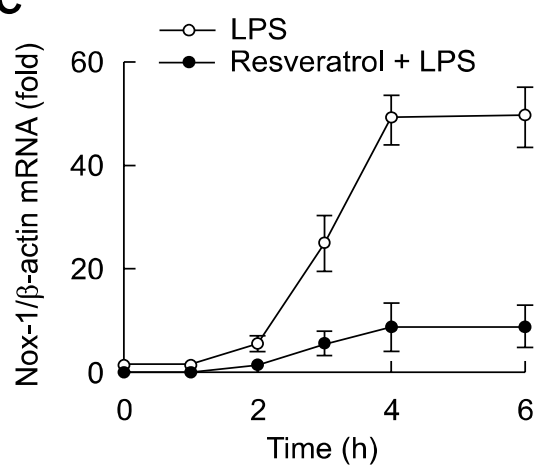

Figure 2. Resveratrol suppress Nox1-mediated ROS generation. (A) Cells were cultured in 96 -well plates, pretreated with resveratrol $(5 \mu \mathrm{M})$, NAC ( $5 \mathrm{mM}$ ) or apocynin $(0.2 \mathrm{mM})$ for $1 \mathrm{~h}$, suspended in PBS and stimulated with LPS containing lucigenin and NADPH. Chemiluminescence was measured in RLU every 10 min over a period of $150 \mathrm{~min}$. $(B, C)$ Macrophages were incubated for $1-6 \mathrm{~h}$ with $100 \mathrm{ng} / \mathrm{ml}$ of LPS in the presence or absence of resveratrol $(5 \mu \mathrm{M})$. At the end of the incubation period, total mRNAs were isolated and Nox1 mRNAs were analyzed by RT-PCR (B) or real-time PCR (C). The mRNA levels of Nox1 were normalized to the levels of $\beta$-actin mRNA.

strongly inhibited the production of ROS induced by LPS (Figure 2A). Next, we have examined the nature of resveratrol-mediated inhibition of ROS production. Since the possibility of Nox participation was suggested in by the observed LPS induced ROS production, we targeted Nox as the molecule that exhibits the ROS blocking effects by resveratrol. The macrophages used in this experiment expressed Nox2 dominantly, but Nox1 was weakly expressed as well (data not shown). LPS treatment increased the expression of Nox 1 mRNA time-dependently (Figure 2B), while having no effects on the expression of Nox2 mRNA (data not shown). Pretreatment of resveratrol inhibited the expression of Nox1 mRNA induced by LPS (Figure 2B). Such effects of resveratrol had similar results in real-time PCR analysis as well (Figure $2 \mathrm{C}$ ).

\section{Resveratrol inhibits LPS-induced MCP-1 expression}

In the process of foam cell formation, MCP-1 is the other well known intermediate factors, so we sought to confirm MCP-1 production induced by LPS through an RT-PCR and ELISA. We examined the effects of resveratrol in the control of MCP-1 production. MCP-1 mRNA expression in Raw264.7 macrophages increased according to the LPS treatment time, with maximum effect noted at $6 \mathrm{~h}$. However, pretreatment with $20 \mu \mathrm{M}$ resveratrol greatly inhibited MCP-1 mRNA expression induced by LPS (Figure 3A). Similar with the results of the conventional RT-PCR, real-time PCR also showed that the MCP-1 mRNA expression induced by LPS was completely inhibited by resveratrol pretreatment (Figure 3B). We quantitatively analyzed the changes in MCP-1 protein production with the culture supernatants using ELISA. MCP-1 protein production induced by LPS decreased depending on the concentration of resveratrol suggesting that MCP-1 expression induced by LPS could be inhibited by resveratrol at the transcription level (Figure $3 \mathrm{C}$ ). In addition, both antioxidants NAC and apocynin also suppressed the LPS-induced MCP-1 production (Figure 3D).

\section{Akt-FoxO3a signaling is a target for the inhibitory effect of resveratrol}

We examined through which signal transduction molecules the production of MCP-1 and the inhibition of Nox1 expression by resveratrol were targeted. Because resveratrol inhibits phosphatidylinositol 3 kinase (PI3K) (Frojdo et al., 2007), we focused on PI3K-Akt signaling based on the description that respiratory burst by resveratrol in human monocytes correlates with the inhibition of $\mathrm{PI}$ KK signaling. To prove that PI3K-Akt signaling controls the expression of MCP-1 and Nox1 expression by LPS, we used the PI3K pathway inhibitor LY294002. LY294002 pretreatment inhibited the expression of LPS-induced MCP-1 and Nox1 mRNA in a concentration dependent manner, and the inhibition of two genes showed similar ten- 
A

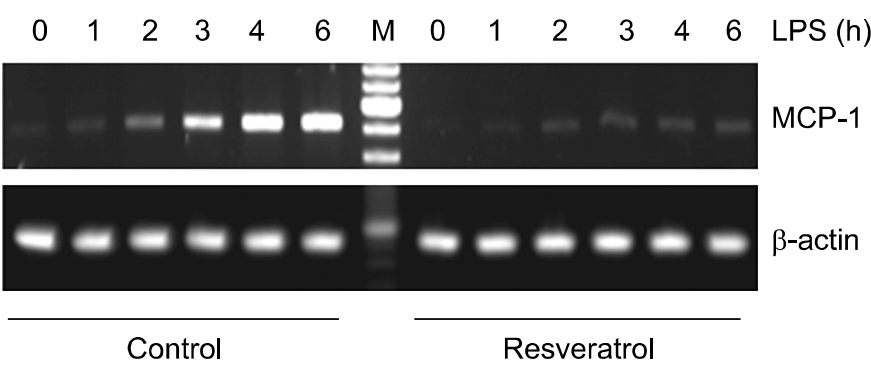

B

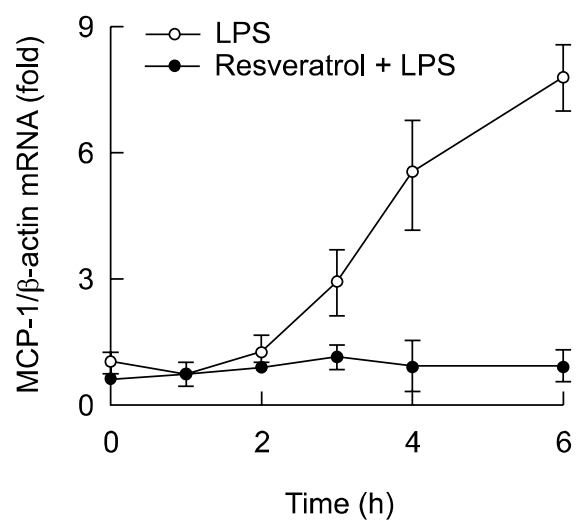

D

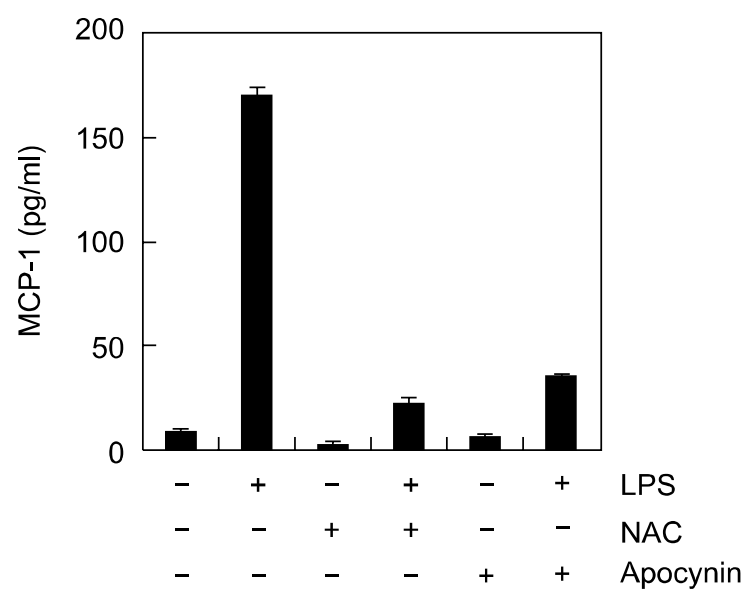

Figure 3. Resveratrol suppress MCP-1 production induced by LPS. (A, B) Cells were treated with a resveratrol $(5 \mu \mathrm{M})$ for $1 \mathrm{~h}$ prior to stimulation with LPS for the indicating times. At the end of the incubation period, total mRNAs were isolated and MCP-1 mRNAs were analyzed by RT-PCR (A) or real-time PCR (B). The mRNA levels of MCP-1 were normalized to the levels of $\beta$-actin mRNA. (C) Cells were pretreated with the indicating concentrations of resveratrol for $1 \mathrm{~h}$ and stimulated with LPS for $6 \mathrm{~h}$. (D) Cells were pretreated with the indicating inhibitors for $1 \mathrm{~h}$ and stimulated with LPS for $6 \mathrm{~h}$. MCP-1 amounts of cell supernatants were analyzed by ELISA.

dencies (Figure $4 \mathrm{~A}$ and $\mathrm{B}$ ). These results suggest that PI3K signaling is essential in the control of LPS-mediated MCP-1 and Nox1 gene expression. LPS treatment maximized the phosphorylation of Akt (the downstream molecule of PI3K) in 20 min and maximized the phosphorylation of ERK1/2 in $30 \mathrm{~min}$ (Figure 4C). While pretreatment of resveratrol did not have much effect in the phosphorylation of ERK $1 / 2$, it significantly decreased phosphorylation of Akt (Figure 4D). These results suggest a possibility that expression inhibition of MCP-1 and Nox1 by resveratrol can be controlled by PI3K-Akt signaling. Various transcription factors are involved in Nox1 regulation. Akt directly phosphorylates FoxO3a, regulating transcriptional activity (Arden and Biggs, 2002). Appropriately, we examined whether LPS could induce phosphorylation of FoxO3a. As shown in Figure 4E, anti-phospho FoxO3a antibody labeled a protein in the extracts of LPS-stimulated cells. Effects of resveratrol on LPS-induced phosphorylation of $\mathrm{FoxO} 3 \mathrm{a}$ were next examined. As shown in Figure $4 \mathrm{~F}$ and 4G, resveratrol or LY294002 suppressed phosphorylation of FoxO3a induced by LPS. These results suggest that PI3K-Akt and transcription factor FoxO3a may be involved in the LPS-induced Nox1 expression (Figure $4 \mathrm{H}$ ).

\section{Discussion}

Elevated ROS is considered a major contributing factor to atherosclerosis, and antioxidants have been found to attenuate ROS effect, which has been suggested to be due primarily to their free radical scavenging activity (Dandona et al., 2007). 
A

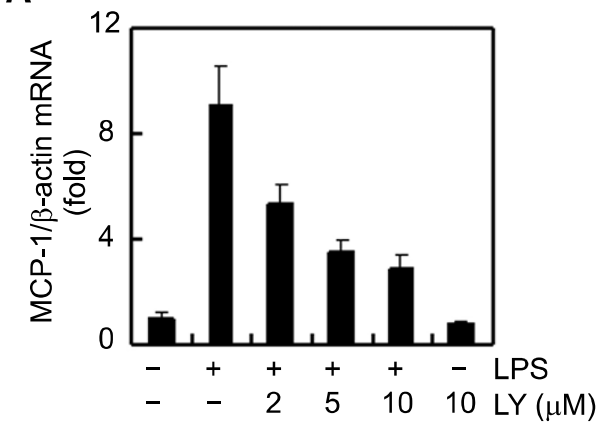

C

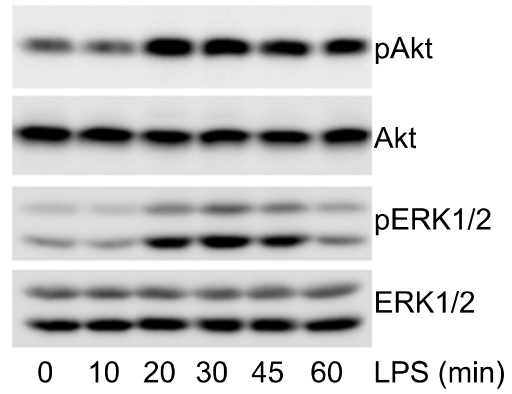

E

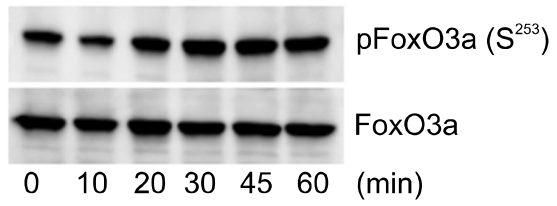

G

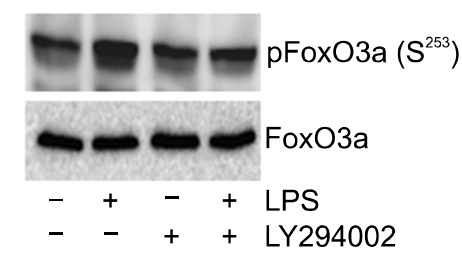

B

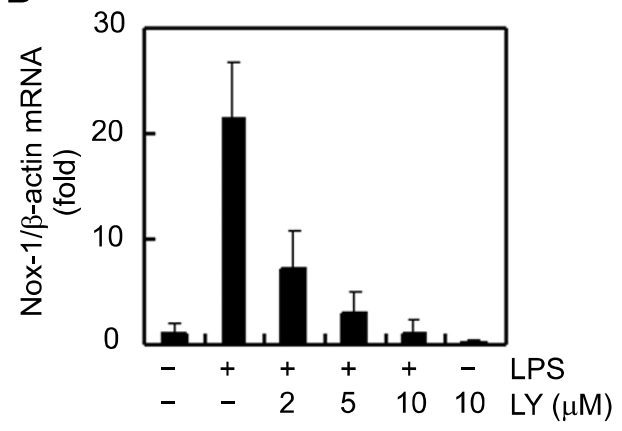

D

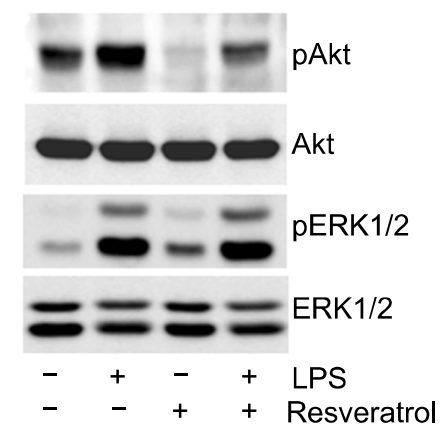

$\mathbf{F}$

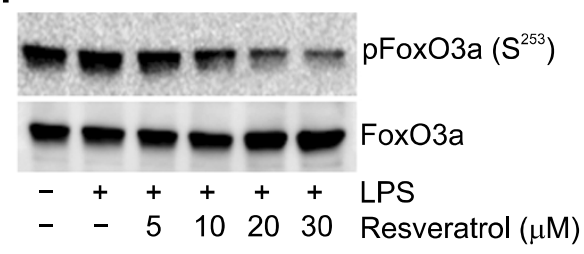

H

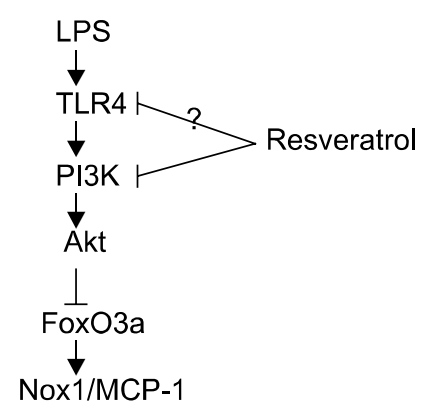

Figure 4. Resveratrol is targeted to the Akt-FoxO3a signaling pathway for Nox1 and MCP-1 expression. (A, B) Cells were treated with an indicating concentrations of LY294002 (PI3K-Akt inhibitor) for $1 \mathrm{~h}$ prior to stimulation with LPS for $6 \mathrm{~h}$. At the end of the incubation time, total mRNAs were isolated and MCP-1 (A) and Nox1 (B) mRNAs were analyzed by real-time PCR. The mRNA levels of MCP-1 and NOX1 were normalized to the levels of $\beta$-actin mRNA. (C) Cells were treated with LPS for the indicated times. After incubation with LPS, cells were lysed and the protein lysates were then analyzed by Western blotting using specific antibodies. (D) Cells were pretreated for $1 \mathrm{~h}$ with resveratrol and then incubated with LPS. After incubation with LPS for 30 min, cells were lysed and the protein lysates were analyzed by Western blotting using specific antibodies. (E) Cells were treated with LPS for the indicated times. After incubation with LPS, cells were lysed and the protein lysates were analyzed by Western blotting using specific anti-FoxO3a or anti-phospho FoxO3a $\left(S^{253}\right)$ antibodies. ( $F$ and $G$ ) Cells were pretreated with the resveratrol or LY294002, and then incubated with LPS. After incubation with LPS for $30 \mathrm{~min}$, cells were lysed and the protein lysates were then analyzed by Western blotting using specific anti-FoxO3a or anti-phospho FoxO3a $\left(\mathrm{S}^{253}\right)$ antibodies. $(\mathrm{H})$ Signaling mechanism involved in LPS-induced Nox1 expression in macrophages and that are targeted by resveratrol.
The present study demonstrates that resveratrol inhibits form cell formation, one of the most important steps in atherosclerosis, as well as ROS production. Especially, resveratrol blocked ROS production by inhibiting the expression of Nox1, and further inhibits MCP-1 production as well. Also, the inhibition of Nox1 expression by resveratrol is accomplished by AktFoxO3a signaling as its target.

Oxidation of LDL favors the transformation of macrophages into foam cells, an important cell involved in atherosclerosis (Vivancos and Moreno, 2008 ), so that modulating its activation process is a potential therapeutic target. The macrophage is thought to play a major role in the pathogenesis of atherosclerosis. This cell differentiates and the resulting macrophage accumulates oxidized LDL to form the characteristic foam cell. Macrophage oxidation of LDL is thought to be dependent on ROS produced from Nox. Presently, we found that resveratrol decreased foam cell formation induced by 
LPS in the $\mu \mathrm{M}$ concentration level, the first such description. Since resveratrol significantly inhibits the expression of Nox1 and blocks ROS production, we think that such inhibition of foam cell formation by resveratrol is accomplished by inhibiting the oxidation of LDL. However, we could not examine the changes in oxidized LDL, and so this suggestion is equivocal.

Resveratrol has been shown to have broad antioxidant activities in a number of biological systems. Resveratrol exerts its effects by decreasing the effect of Nox complex assembly via decreased membrane translocation of gp91 $1^{\text {phox }}$ and Rac1 (Chow et al., 2007). Such results show that the Nox2 system will usually control ROS production because the Nox2 complex in phagocytic cells is a flavocytochrome composed of two membranebound proteins, gp $91^{\text {phox }}$ and $\mathrm{p} 22^{\text {phox }}$, and two cytosolic proteins, $\mathrm{p} 47^{\text {phox }}$ and $\mathrm{p} 67^{\text {phox }}$. The small molecular weight $G$ protein Rac1 is also necessary for the assembly of active Nox2 complex. Resveratrol can lower Nox activity in monocytes contaminated by the intracellular organism Chlamydia pneumonia, which participates in atherosclerosis development; however, this study did not clarify in what type of Nox activation such diminution was mediated. Besides Nox activation, resveratrol inhibits ROS production by inhibiting the activation of enzymes through myeloperoxidase and direct interaction (Kohnen et al., 2007). However, based on our findings, we suggest that the inhibitory effect of resveratrol involves targeting of the Nox1 gene. Even though we could not confirm the effects of resveratrol related to Nox2 activities, we feel confident in suggesting that resveratrol at least controls Nox1 mRNA expression and inhibits ROS production.

Research on Nox has focused on Nox2, but more recent reports have dealt with other Nox species including Nox1. Similar to other Nox species, Nox1 is highly expressed in colon epithelial cells and is principally involved in host defense (Kawahara et al., 2004). ROS production by Nox1 participates in many functions including vasucular smooth muscle cells activation and migration, but Nox1 in the vascular system is usually expressed in smooth muscle cells and Nox2 is usually expressed in macrophages (Bedard and Krause, 2007). Nox2 activation is the most important ROS regulation factor regulating the vascular system, but our data shows that Nox1 expression, especially in macrophages, is an important factor as well. Even though the Nox1 gene is expressed at a low basal level, we think that Nox1 and Nox2 cooperate and regulate ROS production, since expression of the Nox1 gene markedly increases upon exposure to LPS

Despite the evidence for a prominent role of MCP-1 in the development of atherosclerosis, few studies have directly investigated the effect of resveratrol on MCP-1. Moreover, in the process of MCP-1 production related to Nox1, there have not been reports about the effects of resveratrol. ROS is an important factor in controlling MCP-1 production. Indeed, resveratrol-mediated control of MCP-1 production may occur in a disparate fashion. In macrophages, even though resveratrol inhibits various types of inflammatory cytokine productions, it has no effects on MCP-1 (Culpitt et al., 2003). Yet, resveratrol effectively blocks MCP-1 production in human umbilical vein endothelial cells (Cullen et al., 2007b). This disparity could reflect the configuration differences could be from the difference of the trans- and cis-isomers (Leiro et al., 2004). Even though our data was obtained from trans-isomers, resveratrol had strong inhibitory effects in MCP-1 production. Our results show that the inhibition of MCP-1 production by resveratrol could also be achieved by control of Nox1 expression. In the LPS-induced expression of Nox1 and MCP-1, the increased temporal transcription of the two genes was very similar, and the inhibition of the two genes by resveratrol displayed a similar pattern. Our observation that resveratrol inhibited the production of LPS-induced MCP-1 production are entirely consistent with the suggestion that ROS produced by Nox1 activation controls the production of MCP-1.

Presently, we demonstrated that resveratrol blocked LPS-induced Nox1 and MCP-1 expression via inhibition of PI3K-Akt signaling. A previous report demonstrated that resveratrol controls PI3KAkt signaling and affects cell functions as well, with the chemopreventive or chemotherapeutic properties of resveratrol being correlated to its capability to decrease PI3K-controlled phosphorylation of the anti-apoptotic kinase Akt. Resveratrol is a potent class IA PI3K inhibitor and consequently inhibits their downstream signaling molecules (Frojdo et al., 2007). In addition, the inhibitory effect of resveratrol on fMLP-induced ROS production correlates with its inhibitor effect on the PI3K-Akt pathway. Moreover, resveratrol inhibits the activation of Akt and ERK1/2 in mouse cardiac fibroblasts, inhibiting the expression of interleukin-17, which glucose mediates (Venkatachalam et al., 2008). Our results confirmed that the $\mathrm{PI} 3 \mathrm{~K}$ inhibitor inhibits LPS-induced Nox1 and MCP-1 expression, and that resveratrol blocks LPS-induced Akt phosphorylation as well. However, in a finding that differs from previous observations (Venkatachalam et al., 2008), we found that resveratrol did not 
affect ERK1/2 phosphorylation. These present result suggest that in LPS-induced Nox1 and MCP-1 expression, Akt signaling is essential and that resveratrol inhibits Nox1 and MCP-1 production by blocking Akt phosphorylation. But there are suggestions of pleiotropic effects of resveratrol in different types of kinases, and, in fact, there have been reported examples of the regulation of kinase activation (Kundu and Surh, 2004), so further research is necessary.

Several transcription factors may play a role in the expression of Nox1. Previous results suggest that expression of human Nox1 gene is mediated by the GATA-6 transcription factor (Adachi et al., 2008), hepatocyte nuclear factor- $1 \alpha$, and the caudal related homeobox proteins, Cdx1 and Cdx2 (Valente et al., 2008). Interferon- $\gamma$ responsive element and activating transcription factor-1 were also identified in the upstream region of the human Nox1 gene (Katsuyama et al., 2005). On the other hand, there is little information on the exact molecular mechanisms underlying the up-regulation of murine Nox1 gene expression. Of particular our interest is the involvement of FoxO3a, because PI3K-Akt signaling is very important for FoxO3a regulation (Kim et al., 2005). In the present study, we determined the role of resveratrol in FoxO3a phosphorylation induced by LPS. Resveratrol suppressed LPS-induced phosphorylation of FoxO3a in macrophages, implicating FoxO3a as a putative target of resveratrol. Additionally, pretreatment of LY294002, PI3K-Akt pathway inhibitor, also attenuated the LPS-induced FoxO3a phosphorylation. Taken together, these results suggest that resveratrol block the LPS-induced PI3K-Akt pathway and then affected the FoxO3a phosphorylation. However, further investigation is required to deduce whether FoxO3a is responsible for the direct regulation of Nox1 expression.

The ability of resveratrol to inhibit Nox1 and MCP-1 expression may represent an important anti-inflammatory mechanism, which could potentially modulate the activations of important macrophage functions associated with atherosclerosis. Moreover, the ability of resveratrol to modulate the Akt-FoxO3a pathway might represent an important anti-oxidant effect of resveratrol.

\section{Methods}

\section{Reagents and cell culture}

Cell culture reagents, including FBS were obtained from Life Technologies (Grand Island, NY). Antibodies against Akt, phospho-Akt, FoxO3a, and phosphor-FoxO3a $\left(\mathrm{S}^{253}\right)$ were obtained from Cell Signaling Technology (Beverly, MA). A MCP-1 immunoassay kit was obtained from R\&D
Systems (Minneapolis, MN). Escherichia coli LPS, resveratrol, NADPH and lucigenin were obtained from Sigma-Aldrich (St Louis, MO). LY294002 and various inhibitors were purchased from Calbiochem (San Diego, CA). The Raw264.7 macrophage cell line was obtained from the American Type Culture Collection (Manassas, VA).

\section{Lipid uptake assays}

For lipid uptake analysis, macrophages were cultured in 6 -well plates and then treated with LPS and LDL $(50 \mu \mathrm{g} / \mathrm{ml})$ for $24 \mathrm{~h}$. Next, the cells were washed three times with PBS, fixed with $10 \%$ formalin, and then stained with Oil-red $O$. The intracellular lipid droplets were then detected under a DIAPHOT 300 light microscope (Nikon, Tokyo, Japan). The microscopy images were captured with an AxioCam ICcl digital camera system (Carl Zeiss, Jena, Germany).

\section{Western blot analysis}

Macrophages were cultured in 6-well plates and treated with LPS in the presence or absence of an inhibitor. Cell pellets were resuspended in lysis buffer $(50 \mathrm{mM}$ Tris- $\mathrm{HCl}$, pH 8.0, 5 mM EDTA, 150 mM NaCl, 0.5\% Nonidet P-40, 1 mM PMSF, and protease inhibitor cocktail). Proteins were separated by $8 \%$ reducing SDS-PAGE and immunoblotted onto nitrocellulose membranes in $20 \%$ methanol, $25 \mathrm{mM}$ Tris, and $192 \mathrm{mM}$ glycine. Membranes were then blocked with $5 \%$ non-fat dry milk and incubated with primary antibody for $4 \mathrm{~h}$. The membranes were then washed, incubated for $1 \mathrm{~h}$ with secondary antibody conjugated to HRP, rewashed, and finally developed using an enhanced enhanced chemiluminescence system.

\section{Enzyme-linked immunosorbent assay (ELISA)}

Supernatants from treated macrophages were collected and analyzed for MCP-1 using a sandwich ELISA kit (R\&D systems) according to the manufacturer's instructions.

\section{Measurement of ROS generation}

NADPH-dependent ROS formation was measured by monitoring lucigenin-derived chemiluminescence at room temperature using the LMax II luminometer (Molecular Devices, Sunnyvale, CA). Briefly, cells were cultured in 96-well plates, pretreated with inhibitors for $1 \mathrm{~h}$, subsequently suspended in PBS and incubated with lucigenin $(100 \mu \mathrm{M})$ and NADPH $(200 \mu \mathrm{M})$. LPS was added exogenously to the suspended cells. Chemiluminescence was measured in relative light units (RLU) every $10 \mathrm{~min}$ over a period of $150 \mathrm{~min}$.

\section{RT-PCR and real-time PCR}

Total RNA was extracted from cells using the total RNA miniprep kit (Axygen Biosciences, Union City, CA). One microgram of total RNA was used as a template to make first strand cDNA by oligo-dT priming using the Promega reverse transcriptase system (Promega, Madison, WI). 
PCR was then performed using specific primers for target genes, as well as primers for $\beta$-actin as an internal standard. Real-time PCR was performed using a LightCycler 1.5 thermalcycler (Roche Diagnostics, Almere, Netherlands) with SYBR-Green I as the florescent dye, according to the manufacturer's instructions. The cDNA obtained was then amplified using two synthetic primers specific for mouse Nox1 (5'-AAGTGGCTGTACTGGTTGG-3') (5'-GTGAGGAAGAGTCGGTAGTT-3'), mouse MCP-1 (5'-AGAGAGCCAGACGGGAGGAA-3') (5'-GTCACACTGGTCACTCCTAC-3') and mouse $\beta$-actin (5'-AGAGGGAAATCGTGCGTGAC-3') (5'-CAATAGTGATGACCTGGCCGT-3'). The cycling conditions used were $94^{\circ} \mathrm{C}$ for $3 \mathrm{~min}$, followed by 45 cycles of $95^{\circ} \mathrm{C}$ for $5 \mathrm{~s}, 62^{\circ} \mathrm{C}$ for $5 \mathrm{~s}$ and $72^{\circ} \mathrm{C}$ for $12 \mathrm{~s}$.

\section{Acknowledgements}

This work was supported by the Korea Science and Engineering Foundation (KOSEF) grant (R01-2007-000-20087$0)$ and by the Korea Science and Engineering Foundation (KOSEF) grant funded by the Korea government (MEST) (R13-2005-005-01003-0 (2006)).

\section{References}

Adachi Y, Shibai Y, Mitsushita J, Shang WH, Hirose K, Kamata T. Oncogenic Ras upregulates NADPH oxidase 1 gene expression through MEK-ERK-dependent phosphorylation of GATA-6. Oncogene 2008;27:4921-32

Arden KC, Biggs WH 3rd. Regulation of the FoxO family of transcription factors by phosphatidylinositol-3 kinase-activated signaling. Arch Biochem Biophys 2002;403:292-8

Banfi B, Maturana A, Jaconi S, Arnaudeau S, Laforge T, Sinha B, Ligeti E, Demaurex N, Krause KH. A mammalian H+ channel generated through alternative splicing of the NADPH oxidase homolog NOH-1. Science 2000;287:138-42

Bedard K, Krause KH. The NOX family of ROS-generating NADPH oxidases: physiology and pathophysiology. Physiol Rev 2007;87:245-313

Carnevale R, Pignatelli P, Lenti L, Buchetti B, Sanguigni V, Di Santo S, Violi F. LDL are oxidatively modified by platelets via GP91(phox) and accumulate in human monocytes. Faseb J 2007;21:927-34

Chow SE, Hshu YC, Wang JS, Chen JK. Resveratrol attenuates oxLDL-stimulated NADPH oxidase activity and protects endothelial cells from oxidative functional damages. J Appl Physiol 2007;102:1520-7

Cullen JP, Morrow D, Jin Y, von Offenberg Sweeney N, Sitzmann JV, Cahill PA, Redmond EM. Resveratrol inhibits expression and binding activity of the monocyte chemotactic protein-1 receptor, CCR2, on THP-1 monocytes. Atherosclerosis 2007a;195:e125-33

Cullen JP, Morrow D, Jin Y, Curley B, Robinson A, Sitzmann JV, Cahill PA, Redmond EM. Resveratrol, a polyphenolic phytostilbene, inhibits endothelial monocyte chemotactic protein-1 synthesis and secretion. J Vasc Res 2007b;44: 75-84
Culpitt SV, Rogers DF, Fenwick PS, Shah P, De Matos C, Russell RE, Barnes PJ, Donnelly LE. Inhibition by red wine extract, resveratrol, of cytokine release by alveolar macrophages in COPD. Thorax 2003;58:942-6

Dandona P, Ghanim H, Brooks DP. Antioxidant activity of carvedilol in cardiovascular disease. J Hypertens 2007;25: 731-41

Fontaine C, Rigamonti E, Nohara A, Gervois P, Teissier E, Fruchart JC, Staels B, Chinetti-Gbaguidi G. Liver X receptor activation potentiates the lipopolysaccharide response in human macrophages. Circ Res 2007;101:40-9

Frojdo S, Cozzone D, Vidal H, Pirola L. Resveratrol is a class IA phosphoinositide 3-kinase inhibitor. Biochem J 2007;406: 511-8

Gaggioli V, Schwarzer C, Fischer H. Expression of Nox1 in 3T3 cells increases cellular acid production but not proton conductance. Arch Biochem Biophys 2007;459:189-96

Hou X, Xu S, Maitland-Toolan KA, Sato K, Jiang B, Ido Y, Lan F, Walsh K, Wierzbicki M, Verbeuren TJ, Cohen RA, Zang M. SIRT1 regulates hepatocyte lipid metabolism through activating AMP-activated protein kinase. J Biol Chem 2008; 283:20015-26

Katsuyama M, Fan C, Arakawa N, Nishinaka T, Miyagishi M, Taira K, Yabe-Nishimura C. Essential role of ATF-1 in induction of NOX1, a catalytic subunit of NADPH oxidase: involvement of mitochondrial respiratory chain. Biochem J 2005;386:255-61

Kawahara T, Kuwano Y, Teshima-Kondo S, Takeya R, Sumimoto H, Kishi K, Tsunawaki S, Hirayama T, Rokutan K. Role of nicotinamide adenine dinucleotide phosphate oxidase 1 in oxidative burst response to Toll-like receptor 5 signaling in large intestinal epithelial cells. J Immunol 2004;172:3051-8

Kim HS, Skurk C, Maatz H, Shiojima I, Ivashchenko Y, Yoon SW, Park YB, Walsh K. Akt/FOXO3a signaling modulates the endothelial stress response through regulation of heat shock protein 70 expression. Faseb J 2005;19:1042-4

Kohnen S, Franck T, Van Antwerpen P, Boudjeltia KZ, Mouithys-Mickalad A, Deby C, Moguilevsky N, Deby-Dupont G, Lamy M, Serteyn D. Resveratrol inhibits the activity of equine neutrophil myeloperoxidase by a direct interaction with the enzyme. J Agric Food Chem 2007;55:8080-7

Kundu JK, Surh YJ. Molecular basis of chemoprevention by resveratrol: NF-kappaB and AP-1 as potential targets. Mutat Res 2004;555:65-80

Leiro J, Alvarez E, Arranz JA, Laguna R, Uriarte E, Orallo F. Effects of cis-resveratrol on inflammatory murine macrophages: antioxidant activity and down-regulation of inflammatory genes. J Leukoc Biol 2004;75:1156-65

Park CE, Kim MJ, Lee JH, Min BI, Bae H, Choe W, Kim SS, $\mathrm{Ha}$ J. Resveratrol stimulates glucose transport in $\mathrm{C} 2 \mathrm{C} 12$ myotubes by activating AMP-activated protein kinase. Exp Mol Med 2007;39:222-9

Pervaiz S. Resveratrol: from grapevines to mammalian biology. Faseb J 2003;17:1975-85

Schroder K, Helmcke I, Palfi K, Krause KH, Busse R, Brandes RP. Nox1 mediates basic fibroblast growth factor-induced 
migration of vascular smooth muscle cells. Arterioscler Thromb Vasc Biol 2007;27:1736-43

Takeya R, Sumimoto H. Regulation of novel superoxideproducing $\mathrm{NAD}(\mathrm{P}) \mathrm{H}$ oxidases. Antioxid Redox Signal 2006; 8:1523-32

Valente AJ, Zhou Q, Lu Z, He W, Qiang M, Ma W, Li G, Wang L, Banfi B, Steger K, Krause KH, Clark RA, Li S. Regulation of NOX1 expression by GATA, HNF-1alpha, and Cdx transcription factors. Free Radic Biol Med 2008;44:430-43

Vejrazka M, Micek R, Stipek S. Apocynin inhibits NADPH oxidase in phagocytes but stimulates ROS production in non-phagocytic cells. Biochim Biophys Acta 2005;1722: 143-7

Venkatachalam K, Mummidi S, Cortez DM, Prabhu SD,
Valente AJ, Chandrasekar B. Resveratrol inhibits high glucose-induced PI3K/Akt/ERK-dependent interleukin-17 expression in primary mouse cardiac fibroblasts. Am J Physiol Heart Circ Physiol 2008;294:H2078-87

Vivancos M, Moreno JJ. Effect of resveratrol, tyrosol and beta-sitosterol on oxidised low-density lipoprotein-stimulated oxidative stress, arachidonic acid release and prostaglandin E2 synthesis by RAW 264.7 macrophages. Br J Nutr 2008;99:1199-207

Zang M, Xu S, Maitland-Toolan KA, Zuccollo A, Hou X, Jiang $B$, Wierzbicki M, Verbeuren TJ, Cohen RA. Polyphenols stimulate AMP-activated protein kinase, lower lipids, and inhibit accelerated atherosclerosis in diabetic LDL receptordeficient mice. Diabetes 2006;55:2180-91 